This item was submitted to Loughborough's Research Repository by the author.

Items in Figshare are protected by copyright, with all rights reserved, unless otherwise indicated.

\title{
The role of decreasing contact temperatures and skin cooling in the perception of skin wetness
}

PLEASE CITE THE PUBLISHED VERSION

http://dx.doi.org/10.1016/j.neulet.2013.07.015

PUBLISHER

(c) Elsevier Ireland Ltd.

VERSION

AM (Accepted Manuscript)

LICENCE

CC BY-NC-ND 4.0

\section{REPOSITORY RECORD}

Filingeri, Davide, Bernard Redortier, S.G. Hodder, and George Havenith. 2019. "The Role of Decreasing Contact Temperatures and Skin Cooling in the Perception of Skin Wetness". figshare.

https://hdl.handle.net/2134/12887. 
This item was submitted to Loughborough's Institutional Repository (https://dspace.lboro.ac.uk/) by the author and is made available under the following Creative Commons Licence conditions.

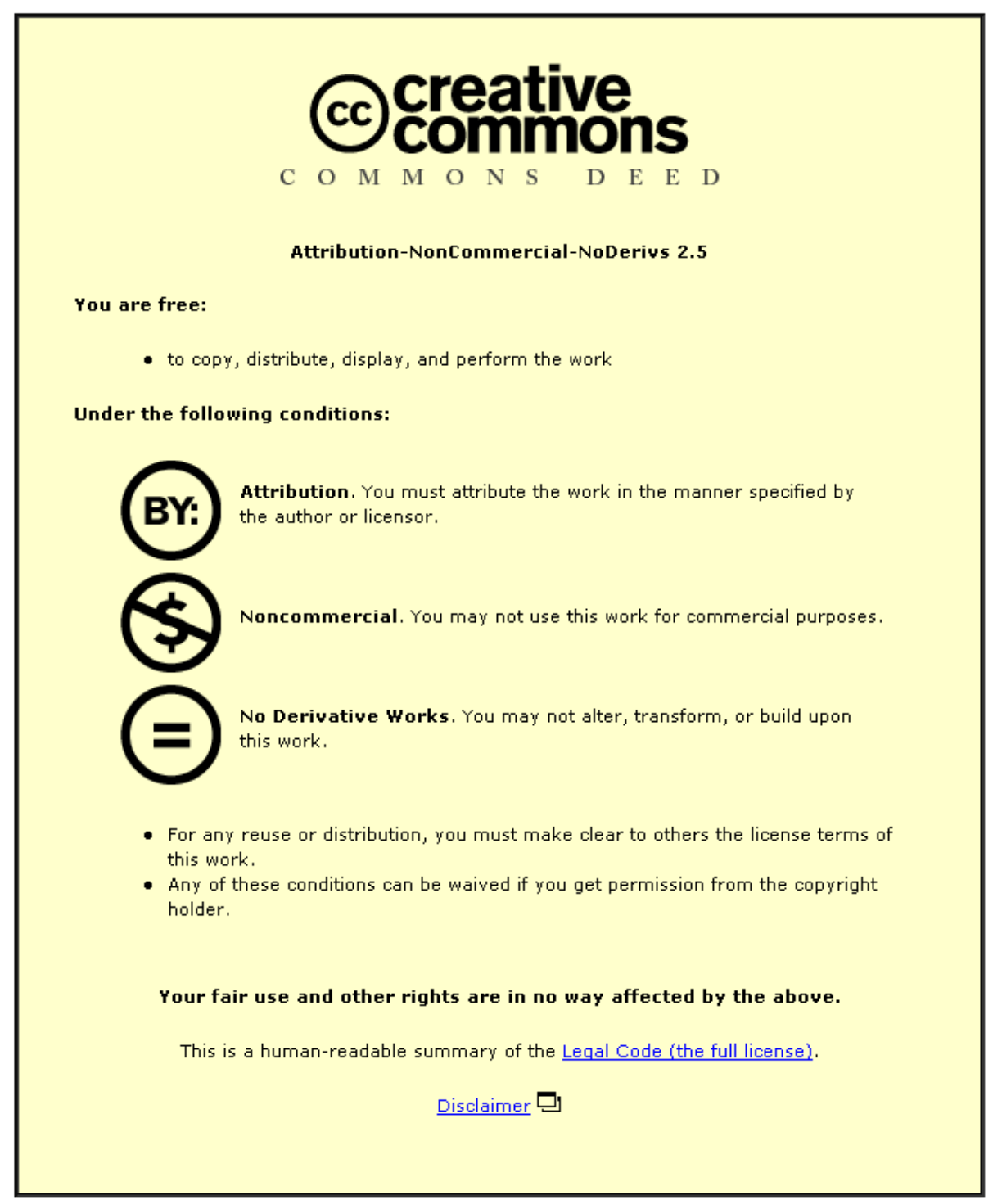

For the full text of this licence, please go to: http://creativecommons.org/licenses/by-nc-nd/2.5/ 


\section{Neuroscience Letters 07/2013}

The role of decreasing contact temperatures and skin cooling in the perception of skin wetness

Davide Filingeria, Bernard Redortier ${ }^{b}$, Simon Hoddera, George Havenitha

a Environmental Ergonomics Research Centre, Loughborough Design School, Loughborough University, Loughborough, LE11 3TU, UK

b Thermal Sciences Laboratory, Oxylane Research, Villeneuve d'Ascq, 59665, France

Corresponding author:

Davide Filingeri, Environmental Ergonomics Research Centre, Loughborough Design School Loughborough University, Loughborough, LE11 3TU, UK, e-mail: D.Filingeri@lboro.ac.uk, phone: +44 (0)1509 223022

E-mails of other authors:

B. Redortier: bernard.redortier@oxylane.com

S. Hodder: S.Hodder@lboro.ac.uk

G. Havenith: G.Havenith@lboro.ac.uk

\section{Abstract}

Cold sensations are suggested as the primary inducer of the perception of skin wetness. However, limited data are available on the effects of skin cooling. Hence, we investigated the role of peripheral cold afferents in the perception of wetness. Six cold-dry stimuli (producing skin cooling rates in a range of 0.02 to $0.41^{\circ} \mathrm{C} / \mathrm{s}$ ) were applied on the forearm of 9 female participants. Skin temperature and conductance, thermal and wetness perception were recorded. Five out of 9 participants perceived wetness as a result of cold-dry stimuli with cooling rates in a range of 0.14 to $0.41^{\circ} \mathrm{C} / \mathrm{s}$, while 4 did not perceive skin wetness at all. Although skin cooling and cold sensations play a role in evoking the perception of wetness, these are not always of a primary importance and other sensory modalities (i.e. touch and vision), as well as the inter-individual variability in thermal sensitivity, might be equally determinant in characterising this perception. Keywords: skin wetness; thermoreceptors; temperature; touch; psychophysics 


\section{Introduction}

Humans interact with their immediate environment through the medium of sensory experiences. However, the way we perceive the world differs qualitatively from the way we sense it [30]. This difference between perception and sensation relies on the fact that our nervous system extracts only certain information from each stimulus and these are then interpreted according to the current situation and previous experiences [21]. Furthermore, perception often results from multisensory experiences as our sensory systems operate within interconnecting, intermodal and cross modal networks [26].

The ability of the central nervous system to combine and process different sensory information into particular perceptions provides the basis for understanding why some of the perceptions we experience are not directly linked to just one specific sensory system. For instance, we experience the perception of "wetness" on the skin though we are not provided with specific receptors for this sensation $[10,22]$. This somatosensory experience is considered a result of the integration of the somatosensory sub-modalities of touch and temperature $[1,3,5,7]$. However, the way in which touch and temperature senses interact to generate the perception of wetness is still unclear $[18,20]$. It has been hypothesised that the activity of thermoreceptors responding to specific drops in skin temperature, such as the ones occurring during the evaporation of sweat from the skin, represents the primary inducer of this perception $[11,18,32]$. Nevertheless, the role played by cold thermoreceptors (i.e. small myelinated $A \delta$ and unmyelinated $C$ fibers) [8] is still unclear and might vary according to the location of these cold sensitive free nerve endings. Indeed, Belmonte and Gallar [2] suggest that the augmented activation of cold thermoreceptors (i.e. corneal trigeminal neurons) located on the human cornea recorded during evaporationinduced ocular surface cooling, seems to be responsible for the perception of ocular dryness. The same physical process (cooling) encoded by the same type of thermoreceptors (cold sensitive) might be therefore primarily responsible for two completely opposite perceptions: dryness and wetness. Furthermore, it could be reasonable hypothesising the interaction of other sensory systems such as vision or touch (in terms of pressure and distribution of pressure) in characterising the perception of wetness [36]. For these reasons, it is still unclear which sensory modality plays the primary input, to what extent, and how it relates with the potentially secondary sensory inputs which overall contribute to characterize wetness as a synthetic perception $[3,24]$. Increasing the knowledge about the neurophysiological bases of the perception of wetness can be useful both for clinical and industrial applications. On the clinical side, it might be used for diagnostic purposes in patients with sensory disorders e.g. diabetic neuropathy $[4,13,25]$. On the industrial side, it might support the development of new strategies in clothing design, as this perception has been shown to play a significant role in the onset of thermal discomfort [15]. 
The present study focuses on the sensation of skin temperature and perception of wetness using a single-blinded psychophysical approach. The aim of the study was to investigate the role of peripheral cold afferents in evoking the perception of skin wetness. Although it has been suggested that this perception can be evoked by the application onto dry skin of a cold-dry stimulus producing a cooling rate of 0.05 to $0.2^{\circ} \mathrm{C} / \mathrm{s}$ [13], no experimental data are currently available involving human participants exposed to different levels of skin cooling. Therefore we investigated a wide range of temperatures, where cold stimuli were applied to the forearm.

\section{Material and methods}

\section{Participants}

Nine healthy female university students $(27.3 \pm 8.8$ years $)$ with no history of sensory-related diseases volunteered to participate in this study. Female participants were preferred to male as they are generally less hairy on the ventral side of the forearm. All participants gave their informed consent for participation. The test procedure and the conditions were explained to each participant. The study design had been approved by the Loughborough University Ethics Committee and testing procedures were in accordance with the tenets of the Declaration of Helsinki.

\section{Design}

The experimental design was based on the application of six cold-dry stimuli of different strength in a balanced order on the bare, left forearm of each participant, while they were resting in an environmental chamber (set at $20^{\circ} \mathrm{C}$ and $50 \%$ relative humidity). Ten minutes were allowed for acclimation and preparation for the test. An s-shaped wooden panel (width: $81 \mathrm{~cm}$; length: $74 \mathrm{~cm}$; height: $60 \mathrm{~cm}$ ) was placed on a table. A hole (width: $12 \mathrm{~cm}$; height: $13 \mathrm{~cm}$ ) in the panel allowed participants to enter their left forearm and lay it down with the palm facing upward. This setup did not allow the participants to see the stimuli that were applied on their forearm. Participants were informed only about the body region subjected to the stimulation. No information was provided on the type and magnitude of the stimulation to limit any expectation effects. To avoid an effect of surprise on the transient cold sensation and wetness perception, a verbal warning was given prior to stimulation during the test. The exact temperatures of cold-dry stimuli were calculated on an individual basis and consisted of a short contact (30 s) with a cold surface set at $-2,-5,-7,-10$, -15 or $-20^{\circ} \mathrm{C}$ than the individual's forearm resting skin temperature [which was recorded using an infrared thermometer (Fluke Corporation, USA)]. The cold-dry stimuli were delivered by a thermal probe (Physitemp Instruments Inc., USA) with a contact surface of $25 \mathrm{~cm}^{2}$ and a weight of $269 \mathrm{~g}$. During the test, participants were asked to maintain their forearm in the required position while the thermal probe was applied to a point corresponding to the mid distance between the elbow and the wrist, on the ventral side. Skin conductance was recorded from the beginning and 
throughout the whole test using the MP35 system (Biopac Systems Inc., USA) which was connected to two electrodes placed on the participant's forearm at a set distance $(7 \mathrm{~cm})$, allowing the thermal probe to be applied in between them. The skin conductance was monitored to estimate sudo-motor activity $[34,35]$ and in the present study was used as a control to establish that no sudo-motor activity occurred i.e. the participant was not sweating due to stress.

\section{Experimental Protocol}

Participants were asked to rate their thermal sensation and wetness perception using psychological rating scales during each of four experimental phases: A) rest; B) cold-dry stimulus; C) bare skin; D) re-warming. In phase A, participants were asked to rate their local thermal sensation and wetness perception at rest without stimulation while forearm skin temperature was recorded with the infrared thermometer. In phase $\mathrm{B}$, the thermal probe (set to the required temperature) was applied to the forearm and left in full contact with the skin site for $30 \mathrm{~s}$, while participants were asked to rate their local thermal sensation and wetness perception $10 \mathrm{~s}$ after the application. The probe was then removed and the skin temperature was immediately recorded. The skin site was left bare for $30 \mathrm{~s}$ (phase $\mathrm{C}$ ). At the end of this phase participants were asked to rate their local thermal sensation and wetness perception, and skin temperature was again recorded. Finally (phase D - re-warming), the thermal probe was set at a temperature corresponding to the one recorded at the beginning of the test (the individual's baseline) and then applied for $30 \mathrm{~s}$ to re-warm the skin. Participants were then asked to rate their thermal sensation and wetness perception for the last time and skin temperature was recorded immediately after the thermal probe was removed. This sequence was repeated for each stimulus allowing at least one minute in between. Each participant had only one presentation of each stimulus. The order of the stimuli was balanced within and between the tests to avoid any order effect.

\section{Psychological rating scales}

We designed three psychological rating scales to record individual thermal sensation and wetness perception [29]. An 11 point thermal sensation scale ( -5 extremely cold; -4 very cold; -3 cold; -2 cool; -1 slightly cool; 0 neutral; +1 slightly warm; +2 warm; +3 hot; +4 very hot; +5 extremely hot) was used at rest and during the re-warming; a seven points thermal sensation scale [from 0 to 6 , where 0 was labelled as not cold at all and 6 as extremely cold (with no labels in between them)] was used during both cold stimulus and bare skin phases. Finally, a seven point wetness perception scale [from 0 to 6 , where 0 was labelled as dry and 6 as extremely wet (with no labels in between them)] was used during all the phases of each test. We defined the value " 1 " of the scale as our set threshold to identify a clearly perceived wetness. Participants familiarised with the scales during the acclimation period. During the experimental protocol, participants rated verbally their sensations, which were immediately recorded by the investigator. 


\section{Statistical Analysis}

In the present study, the independent variable was the temperature of the thermal probe (the relative cold stimulus based on the individual baseline skin temperature) and the dependent variables were the forearm skin temperature, skin conductance, thermal sensation and wetness perception. Data were tested for normality of distribution using Shapiro-Wilk test. Skin temperature data were analysed by a one way repeated measures analysis of variance. (ANOVA) Post-hoc analyses using a Tukey's test were performed to account for multiple comparisons and sample size effect.

Thermal and wetness ratings were analysed using a Friedman test (non-parametric randomized block ANOVA) and post-hoc analyses were performed using a Wilcoxon signed rank tests. Huynh-Feldt, Geisser-Greenhouse, and lower bound corrections were undertaken to adjust the degrees of freedom for the averaged tests of significance. A linear regression analysis was performed to assess the relationship between the variation in skin temperature from baseline and the relative cold stimuli. Ordinal regression analyses were performed between the thermal and wetness ratings and the relative cold stimuli. Finally, a Spearman's rank correlation coefficient was calculated to investigate the degree of association between thermal sensation and wetness perception. All data were analysed using SPSS Statistics 19 (IBM, Armonk, NY) and were reported as means \pm standard deviation (SD). In all analyses, $p<0.05$ was used to establish significant differences.

\section{Results}

\section{Skin temperature}

Skin temperature data were normally distributed and were thus analysed by a repeated measure ANOVA and Tukey's test. The resting skin temperature before stimulation $\left(29.7 \pm 1.4{ }^{\circ} \mathrm{C}\right)$ did not significantly differ between each of the six conditions $(p>0.05)$ confirming the effectiveness of the balanced order of the stimuli in avoiding any order effect. Furthermore, no differences were recorded in the post re-warming skin temperature $\left(29.5 \pm 1.2^{\circ} \mathrm{C}\right)$ between conditions $(p>0.05)$, confirming that the skin was effectively re-warmed to the resting value.

During the stimulation, each cold-dry stimulus produced significantly different decreases in the skin temperature $\left(F=71.61_{(2.32,18.57)}, p<0.001\right)$ varying in a range between $-0.8 \pm 0.8^{\circ} \mathrm{C}$ to -12.3 $\pm 2.7^{\circ} \mathrm{C}$ from the baseline skin temperature, corresponding to a cooling rate range of $0.02 \pm$ $0.02^{\circ} \mathrm{C} / \mathrm{s}$ to $0.41 \pm 0.09^{\circ} \mathrm{C} / \mathrm{s}$ (fig. $1 \mathrm{a}$ ).

\section{Thermal sensation and wetness perception}

Thermal sensation and wetness perception data were analysed by a Friedman test and Wilcoxon signed rank tests. Resting thermal sensation and wetness perception did not significantly differ 
between the six conditions ( $p>0.05)$ with an average score of $-0.2 \pm 0.2$ and $0.2 \pm 0.1$ respectively. Furthermore, no differences were found during the re-warming phase of each condition ( $p>0.05$ ), as shown by a recorded average thermal sensation of $+1.4 \pm 0.2$ and an average wetness perception of $0.2 \pm 0.2$.

Stimuli produced statistically significant differences $\left(X^{2}=34.7_{(5,9)}, p<0.001\right)$ in thermal sensation both during stimulation (varying in a range between $0.7 \pm 1$ to $4.1 \pm 1.8$ ) as well as during the bare skin phase (varying in a range between $0.8 \pm 1.1$ to $2.3 \pm 1.1$ ). Data related to wetness perception showed that overall, in 19 out of 54 scores (35\%) recorded during phase B (cold-dry stimulation), a cold-dry stimulus was perceived as cold-wet. We then proceeded with the analysis of individual data which showed the existence of two sub-groups within the whole sample tested in this experiment. Indeed, five out of nine participants reported wetness perceptions varying significantly according to the rate of skin cooling, either during the cold-dry stimulation and the following bare skin phase, whereas four out of nine participants did not perceive wetness at all. At this point we decided to identify the two groups as "responders" and "non-responders" [9] to the cold-dry stimuli we used in this study and thus performing a separate analysis in terms of wetness perception.

Data related to the responders group showed statistically significant differences $\left(X^{2}=16.2_{(5,5)}\right.$, $p<0.01)$ in the wetness perception scored during both the cold-dry stimulation and the bare skin phase (fig. 1b), with the threshold we set (point "1" of the scale) to identify a clearly perceived wetness reached during four out of the six conditions $\left(-7,-10,-15\right.$ and $-20^{\circ} \mathrm{C}$ respectively).

\section{Regression and correlation analysis}

The relationship between the variation in skin temperature from baseline and the relative cold stimuli (assessed by a linear regression analysis which included data from the whole sample) was found to be statistically significant $\left(p<0.001 ; R^{2}=0.83\right.$; regression coefficient $b_{0}=0.605$; regression coefficient $b_{1}=0.632$ ). Similarly, the relationship between the thermal ratings and the relative cold stimuli (assessed by an ordinal regression analysis which included data from the whole sample) was found to be statistically significant [ $\rho<0.001$; Chi-square analysis (Pearson; Deviance): $p>0.05$; Nagelkerke (pseudo $\left.R^{2}\right)=0.58$; Test of parallel lines: $p>0.05$ ]. The relationship between the wetness ratings and the relative cold stimuli (assessed by an ordinal regression analysis which included only the data from the responders sub-group) was also found to be statistically significant [ $p<0.001$; Chi-square analysis (Pearson; Deviance): $p>0.05$; Nagelkerke (pseudo $\mathrm{R}^{2}$ ) $=0.57$; Test of parallel lines: $p>0.05$ ]. Finally, the degree of association between thermal sensation and wetness perception (assessed by a Spearman's rank correlation test which included only the data from the responders sub-group) was found to be statistically significant $(p<0.001$; Spearman's rho $=0.78)$. 


\section{Skin conductance}

Average values did not significantly change during testing procedures and were observed to remain constantly at a level below $0.5 \mu \mathrm{S}$. These results confirm that no variations in sudo-motor activity occurred during the experiment.

\section{Discussion}

The aim of this study was to investigate the mechanisms responsible for the perception of skin wetness with regard to cold temperature sensing. The experimental protocol was designed to ensure that a dry skin site would be exposed for a relatively short time to a wide range of local cold-dry stimuli. This approach resulted in evoking artificial wetness perceptions, with $35 \%$ of the cold-dry stimuli applied on the participants' forearms being perceived as cold-wet.

This first outcome showed that the wetness perception did relate to the activation of the thermal afferents responding to skin cooling. However, this was true only for a sub-group of five participants. Data from this sub-group seem aligned to the findings of Daanen [13] who measured the temperature course of the skin (i.e. temperature' $s$ drop of 1 to $5^{\circ} \mathrm{C}$ with a 0.05 to $0.2^{\circ} \mathrm{C} / \mathrm{s}$ cooling rate) when this was wetted with drops of water with volumes in a range of 0.01 to $0.1 \mathrm{ml}$. The author suggested that the cold sensations experienced when such skin cooling occurs can contribute to the perception of skin wetness. Therefore, exposing the skin to a colddry stimulus producing such skin cooling was hypothesised to evoke an illusory perception of skin wetness. In our study, this hypothesis was confirmed, as when the application of cold-dry stimuli produced a drop in skin temperature ranging between 1.4 and $4.1^{\circ} \mathrm{C}$ with a cooling rate of 0.14 to $0.41^{\circ} \mathrm{C} / \mathrm{s}$, a clear wetness perception was evoked, whereas when the cold-dry stimulation produced a drop in skin temperature of 0.2 to $0.7^{\circ} \mathrm{C}$ with a cooling rate of 0.02 to $0.07^{\circ} \mathrm{C} / \mathrm{s}$, wetness was little evoked and decreasing thermal sensations prevailed.

Therefore we suggest that, the rate of heat transfer from the skin to a colder surface seems to play a significant role not only in thermal and touch discrimination of different materials [4] but also in characterising the perception of a cold stimulus as simply cold or as also wet. During our experimental conditions a skin cooling rate threshold for the perception of "cold-dryness" and "cold-wetness" was identified (i.e. between 0.07 and $0.14^{\circ} \mathrm{C} / \mathrm{s}$ ) and further evidence has been added to the work of Daanen [13], as we observed that greater skin cooling rates (up to $0.41^{\circ} \mathrm{C} / \mathrm{s}$ ) than the one proposed by the author $\left(0.05\right.$ to $\left.0.2^{\circ} \mathrm{C} / \mathrm{s}\right)$, can also contribute to evoke a wetness perception.

However, although at this point it might be proposed that skin cooling and thus temperature sensations alone might be sufficient to generate the perception of skin wetness, \{as suggested by Bergmann Tiest et al. [6] in their recent work in which phase-change materials inducing cool sensations were perceived as wet\}, the presence of a non-responders sub-group within the 
whole sample, who did not perceive wetness during any of the experimental conditions, contrasts with this conclusion. A possible explanation of the incongruent sensory perceptions recorded in the two sub-groups might be related the properties of the stimulus, which were voluntarily limited to focus on the effects produced by skin cooling. The lack of intra- and inter-sensory interaction, particularly in terms of touch and vision (the probe was applied but not moved and participants could not see the stimulation area), might be primary responsible for the heterogeneity of the responses. Indeed, it has been shown that the co-activity of highly specialised receptors with different individual properties is essential in generating the variety of cutaneous sensations we encounter in everyday life, particularly in complex perceptions such as skin wetness $[1,26]$. Thus, the role of the other somatosensory sub-modalities might be equally as important as the skin cooling itself [1], which can therefore not always be sufficient in evoking the perception of wetness. In the work of Bergmann Tiest et al. [6], no non-responders group was identified, a fact which might be the reason why the author concluded that touch-related sensations seem unnecessary and thermal sensations can be sufficient in evoking the perception of skin wetness. However, it has to be observed that in the mentioned work, participant where asked to choose which one felt wetter between a treated (with phase-change materials) and an untreated fabric. In our view, this experimental approach affected the participants' responses as no option of reporting the absence of wetness was given to them. In principle, if both samples had been experienced as dry, the lower score observable in the group would have been a $50 \%$, which means that neither in that case a non-responders subgroup would have been identified.

Therefore, although decreases in skin temperature may sometimes be sufficient, a more complex sensory-blending hypothesis should be considered to explain the psycho-physiological process responsible of the perception of skin wetness $[18,20]$. Studies by Gerrett [16] and everyday experience suggest that we are able to perceive the wetness even when the skin temperature does not decrease (e.g. during exposure to hot environmental conditions or when in contact with hot water). Furthermore, our group recently observed that the individual ability to discriminate between dryness and wetness is limited during the static contact with a warm dry or wet surface as no skin cooling (and thus no cold sensations) occurs [14]. Thus, defining some particular activations of the cold afferents as sufficient to generate this perception (regardless of other sensory interactions [6]) might be limiting in the light of the complex interconnecting, intermodal and cross modal networks our sensory systems operate within [26].

The way we perceive "feelings" from our body results from complex integrations between the activity of the exteroceptive and interoceptive systems [11]. Furthermore, converging evidence suggests a phylogenetically new system (which integrates information about the overall homeostatic condition of the body) as one of the principal neuroanatomical structures that differentiate humans from non-human primates [12]. This hypothesis confirms the multimodal as one of the most appropriate approaches when investigating the mechanisms of sensory 
integration. As the perception of skin wetness represents one of the numerous somatosensory experiences that allow us to sense and perceive our immediate environment (and eventually interact with it) [26], it is reasonable to hypothesise that other sensory inputs than just temperature (i.e. touch, vision) can significantly influence the way we experience this complex perception.

Finally, although the neurological and molecular basis of thermal sensations have been largely investigated and described [28,31,33], individual thermal sensations are much more difficult to predict due to other parameters relating to wider and more complex relationships between physiological and psychological responses $[23,27]$. For instance, the inter-individual variability is a critical factor in determining the psychological responses resulting from somatic stimulation, as shown in the role played by individual characteristics such as gender, age, ethnicity and physical fitness in influencing the cutaneous thermal thresholds and thus the variability of thermal sensations [19,23].

\section{Conclusion}

In this study we found that skin cooling and thermal sensations can contribute significantly to the perception of skin wetness. We have shown that a cooling rate threshold for a cold stimulus to be perceived as wet is identifiable based on the rate of heat transfer from the skin. Also, greater cooling rates than the ones currently proposed, were shown to evoke wetness perceptions. However, the activity of peripheral cold afferents as a result of skin cooling has been shown to not always be sufficient in evoking the perception of wetness. This suggests that the intra- and inter-sensory interaction with other modalities (i.e. touch, vision), as well as the inter-individual variability, might have a role as equally determinant as the one played by the temperature sense in affecting individual thresholds for the perception of complex somatosensory experiences such as skin wetness. Little is known about the temperature sensing system across the body and even less is known on how this specifically interacts with the other sensory systems to produce the variety of somatosensory perceptions we experience every day.

\section{Role of funding body}

The present research was done in the context of an industry co-funded $\mathrm{PhD}$.

\section{Acknowledgments}

The authors would like to thank $\mathrm{Dr}$ Rochelle Ackerley for her constructive comments and insightful discussions, and Victoria Kendrick for proof reading the manuscript.

\section{References}


[1] R. Ackerley, H. Olausson, J. Wessberg, F. McGlone, Wetness perception across body sites, Neurosci. Lett. 522 (2012) 73-77.

[2] C. Belmonte, J. Gallar, Cold thermoreceptors, unexpected players in tear production and ocular dryness sensations, Invest. Ophth. Vis. Sci. 52 (2011) 3888-3892.

[3] I. Bentley, The synthetic experiment, Am. J. Psychol. 11 (1900) 405-425.

[4] W.M. Bergmann Tiest, A.M.L. Kappers, Tacaile perception of thermal diffusivity, Atten. Percep. Psychophys. 71 (2009) 481-489.

[5] W.M. Bergmann Tiest, N.D. Kosters, A.M.L. Kappers, H. A. M. Daanen, Haptic perception of wetness, Acta Psychol. 141 (2012) 159-163.

[6] W.M. Bergmann Tiest, N.D. Kosters, A.M.L. Kappers, H. A. M. Daanen, Phase change materials and the perception of wetness, Ergonomics 55 (2012) 508-512.

[7] S.J. Bolanowski, G.A. Gescheider, A.M. Fontana, J.L. Niemec, J.. Troblay, The effects of heat-induced pain on the detectability, discriminability, and sensation magnitude of vibrotactile stimuli, Somatosens. Mot Res. 18 (2001) 5-9.

[8] M. Campero, H. Bostock, Unmyelinated afferents in human skin and their responsiveness to low temperature, Neurosci. Lett. 470 (2010) 188-192.

[9] J.R. Carter, C.A. Ray, Sympathetic neural responses to mental stress: responders, nonresponders and sex differences, Am. J. Physiol. Heart C. 296 (2009) H847-853.

[10] R. Clark, O. Edholm, Man and his thermal environment, Arnold, London, 1985.

[11] A. Craig, Interoception: the sense of the physiological condition of the body, Curr. Opin. Neurobiol. 13 (2003) 500-505.

[12] A. Craig, How do you feel? Interoception: the sense of the physiological condition of the body, Nat. Neurosci. 3 (2002) 655-666.

[13] H. A. M. Daanen, Method and system for alerting the occurrence of wetness, EP Patent 2,110,108, 2009.

[14] D. Filingeri, B. Redortier, S. Hodder, G. Havenith, Individual ability to discriminate between wetness and dryness during short contacts with a warm surface, in: J.D. Cotter, 
S.J.E. Lucas, T. Mundel (Eds.), 15th International Conference on Environmental Ergonomics (ICEE), 2013: p. 180.

[15] T. Fukazawa, G. Havenith, Differences in comfort perception in relation to local and whole body skin wettedness, Eur. J. Appl. Physiol. 106 (2009) 15-24.

[16] N. Gerrett, Body mapping of perceptual responses to sweat and warm stimuli and their relation to physiological parameters, Unpublished doctoral dissertation, Loughborough University, UK (2012).

[17] H. Gin, R. Baudoin, C.H. Raffaitin, V. Rigalleau, C. Gonzalez, Non-invasive and quantitative assessment of sudomotor function for peripheral diabetic neuropathy evaluation, Diabetes Metab. 37 (2011) 527-532.

[18] S. Guest, C. Catmur, D. Lloyd, C. Spence, Audiotactile interactions in roughness perception, Exp. Brain Res. 146 (2002) 161-171.

[19] G. Havenith, The relative influence of physical fitness, acclimatization state, anthropometric measures and gender on individual reactions to heat stress, Eur. J. Appl. Physiol. 1 (1990) 419-427.

[20] V. Jousmäki, R. Hari, Parchment-skin illusion: sound-biased touch, Curr. Biol. 8 (1998) 190-191.

[21] E.R. Kandel, J.H. Schwartz, T.M. Jessell, Principles of neural sciences, fourth ed., McGraw-Hill, USA, 2000.

[22] J. Lee, K. Nakao, Y. Tochihara, Validity of perceived skin wettedness mapping to evaluate heat strain, Eur. J. Appl. Physiol. 111 (2011) 2581-2591.

[23] J. Lee, M. Saat, C. Chou, N. Hashiguchi, T. Wijayanto, H. Wakabayashi, Y. Tochihara, Cutaneous warm and cool sensation thresholds and the inter-threshold zone in Malaysian and Japanese males, J. Therm. Biol. 35 (2010) 70-76.

[24] Y. Li, Perceptions of temperature, moisture and comfort in clothing during environmental transients, Ergonomics 48 (2005) 234-248.

[25] T. Mano, S. Iwase, S. Toma, Microneurography as a tool in clinical neurophysiology to investigate peripheral neural traffic in humans, Clin. Neurophysiol. 117 (2006) 2357-2384. 
[26] F. McGlone, D. Reilly, The cutaneous sensory system, Neurosci. Biobehav. R. 34 (2010) 148-159.

[27] D. D. McKemy, How cold is it? TRPM8 and TRPA1 in the molecular logic of cold sensation, Mol. Pain 1 (2005) 16.

[28] D. D. McKemy, The molecular and cellular basis of cold sensation, ACS Chem. Neurosci. 4 (2013) 238-247.

[29] B. Olesen, G. Brager, A better way to predict comfort: The new ASHRAE standard 552004, ASHRAE J. 8 (2004).

[30] L. M. Parsons, S. Shimojo, Perceived spatial organization of cutaneous patterns on surfaces of the human body in various positions, J. Exp. Psychol. Human 13 (1987) 488504.

[31] R. Schepers, M. Ringkamp, Thermoreceptors and thermosensitive afferents, Neurosci. Biobehav. R. 34 (2010) 177-184.

[32] G. Storaas, K. Bakkevig, Correlation between measured skin wettedness and subjective sensations of skin wetness, in: The 7th International Conference on Environmental Ergonomics (ICEE), 1996: pp. 131-134.

[33] M. Tominaga, M. Caterina, Thermosensation and pain, J. Neurobiol. 61 (2004) 3-12.

[34] C. Tronstad, G.E. Gjein, S. Grimnes, Ø.G. Martinsen, A.-L. Krogstad, E. Fosse, Electrical measurement of sweat activity, Physiol. Meas. 29 (2008) S407-415.

[35] R. Vetrugno, R. Liguori, P. Cortelli, P. Montagna, Sympathetic skin response: basic mechanisms and clinical applications, Clin. Auton. Res. 13 (2003) 256-270.

[36] Z. Wang, Y. Li, Y.L. Kowk, C.Y. Yeung, Mathematical simulation of the perception of fabric thermal and moisture sensations, Text. Res. J. 72 (2002) 327-334. 
Figure 1: (a) Relative variations in skin temperature drop from baseline $\left(\Delta T_{s k}\right)$ and corresponding cooling rates as a result of each of the six cold-dry stimuli. (b) Wetness perception scores recorded in the responders sub-group as a result of each of the six cold-dry stimuli (phase B) and during the following bare skin phase $(C)\left({ }^{*} p<0.05\right)$. Skin cooling rates corresponding to each stimulus are reported between brackets. The point "1" of the wetness perception scale corresponds to the threshold set to identify perceived skin wetness.

\section{(a)}

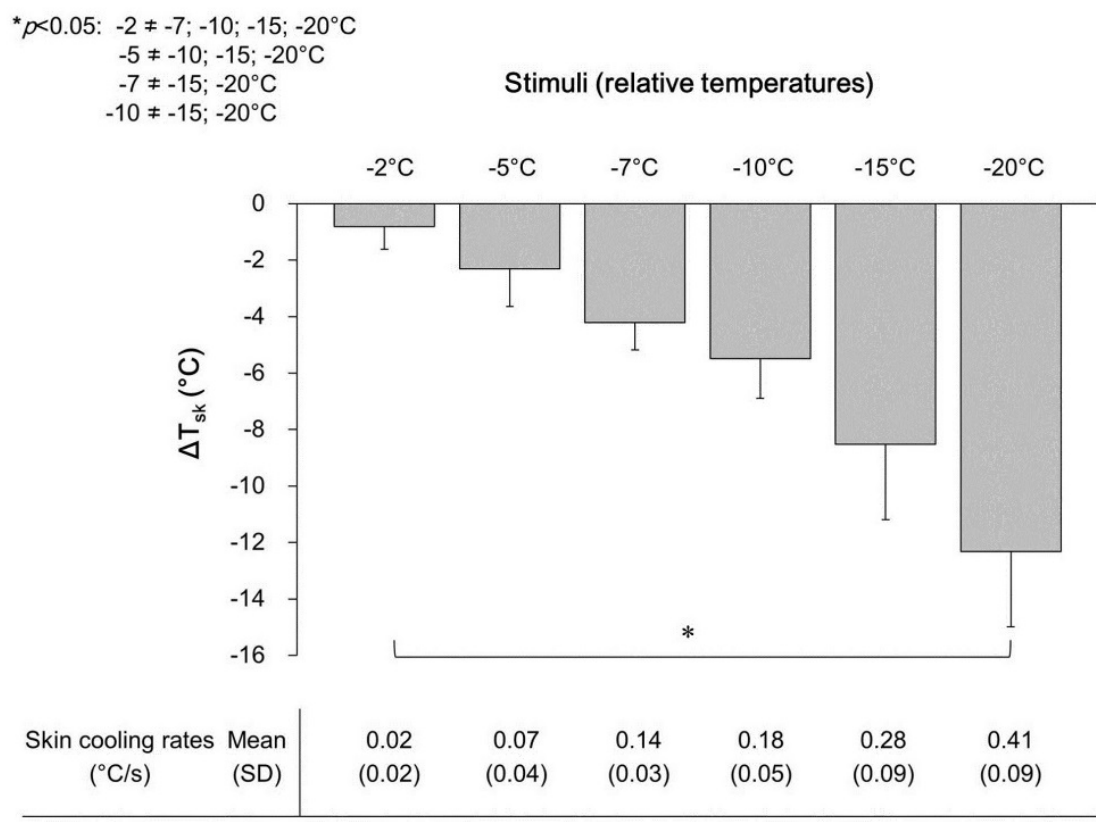

(b)

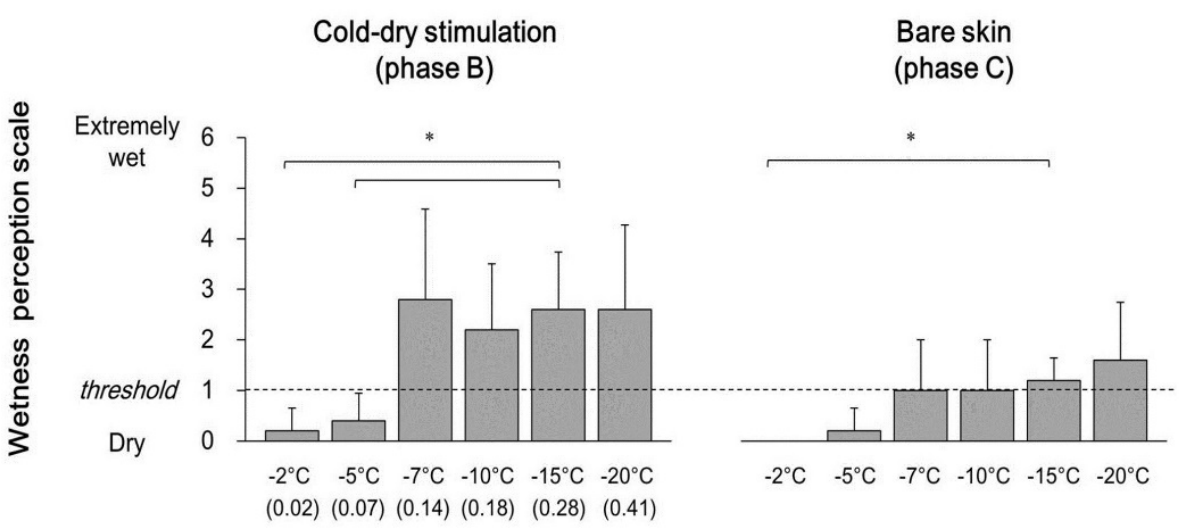

